\title{
Scinderin promotes the invasion and metastasis of gastric cancer cells and predicts the outcome of patients
}

Authors: Jia-jia Liu ${ }^{\text {a,1 }}$, Jun-yan Liu ${ }^{\text {a,1 }}$, Jun Chen ${ }^{\text {a }}$, Yi-xi Wu ${ }^{\text {a }}$, Peng Yan ${ }^{\text {a }}$ Cheng-dong Ji ${ }^{\mathrm{b}}$, Yan-xia Wang ${ }^{\mathrm{b}}$, Dong-fang Xiang ${ }^{\mathrm{b}}$, Xia Zhang ${ }^{\mathrm{b}}$, Peng Zhang ${ }^{\mathrm{b}}$, You-hong Cui ${ }^{b}$, Ji Ming Wang ${ }^{\text {b, c }}$, Xiu-wu Bian ${ }^{\text {b,* }}$, Feng Qian ${ }^{\text {a,* }}$

${ }^{a}$ Department of General Surgery and Center of Minimal Invasive Gastrointestinal Surgery, Southwest Hospital, Third Military Medical University, Chongqing 400038, China.

${ }^{\mathrm{b}}$ Institute of Pathology and Southwest Cancer Center, Southwest Hospital, Third Military Medical University, and Key Laboratory of Tumor Immunopathology of Ministry of Education of China, Chongqing 400038, China.

${ }^{\mathrm{c}}$ Cancer and Inflammation Program, Center for Cancer Research, National Cancer Institute, Frederick, MD 21702, USA.

${ }^{*}$ Correspondence to: Feng Qian, M.D. and Xiu-wu Bian, M.D., Ph.D. Tel.: +86 23 68765775 (Qian) and +86 2368764431 (Bian). Fax: +86 2368754167 (Qian) and +862368754431 (Bian). E-mail address: qianfengpuwaia@ 163.com (Qian) and bianxiuwu@263.net (Bian).

${ }^{1}$ These authors contributed equally to the study. 


\title{
Highlights:
}

- High levels of SCIN expression in GC tissue predict a poor outcome in patients.

- SCIN enhances the invasive and metastatic capabilities of GC cells in vitro and in vivo.

- Filopodium formation is involved in the SCIN- promoted invasion and metastasis of GC cells.

- Suppression of SCIN in GC cells is accompanied by decreased expression of Cdc42, an important regulator of filopodia.

\begin{abstract}
Invasion and metastasis are major malignant characteristics of human gastric cancer (GC), but the underlying molecular mechanisms are poorly understood. Recent studies have shown that scinderin (SCIN), an actin severing and capping protein that regulates the actin cytoskeleton, is involved in the proliferation and migration of certain cancer cells. Accordingly, this study aimed to investigate the potential role of SCIN in the invasion and metastasis of human GC cells and to evaluate its prognostic value for GC patients. We found that high levels of SCIN expression in GC tumors was correlated with poor overall survival of patients. Silencing of SCIN effectively suppressed the migratory and invasive capabilities of human GC cells in vitro and tumorigenicity and metastasis in vivo. Furthermore, knockdown of SCIN markedly inhibited the formation of filopodia, decreasing GC cell migration and the expression
\end{abstract}


of $\mathrm{Cdc} 42$, an important regulator of filopodia by GC cells. These findings suggest that SCIN may be a novel prognostic marker and a potential therapeutic target in human GC.

Key words: scinderin; gastric cancer; invasion; metastasis; filopodia; Cdc42.

\section{Introduction}

Gastric cancer (GC) is the fourth-most prevalent cancer worldwide and the second-most common cause of cancer-related death [1]. More than $70 \%$ of new GC cases and deaths occur in developing countries, particularly in eastern Asia [2]. Although the mortality rate has been decreasing in recent years due to significant improvements in early diagnosis, surgical techniques and chemotherapy, the long-term survival rate for patients with advanced-stage GC remains low. As invasion and metastasis are important properties of GC contributing to poor patient prognosis [3], a better understanding of the molecular mechanisms underlying GC cell invasion and metastasis is crucial for developing effective treatments.

Cancer cell invasion and metastasis are closely related to cell migration, a process that involves actin filament dynamics regulated by various actin-binding proteins [4-6]. Scinderin (SCIN), also known as adseverin, is a major member of the actin-binding protein family that regulates actin filament reconstruction by severing pre-existing actin filaments, capping filament ends and nucleating actin polymerization from 
monomers in a $\mathrm{Ca}_{2}^{+}$-dependent manner [7-8]. SCIN is mainly expressed by endocrine and secretory cells and plays key roles in vesicle transport and exocytosis by organizing the cytoskeleton underneath the plasma membrane [9-10]. In addition, SCIN has been suggested to be a multifunctional molecule that regulates other cellular functions such as osteoclastogenesis and osteoclast function [11-13], the differentiation of megakaryoblasts [14], and the proliferation, migration and odontoblastic differentiation of dental pulp cells [15]. Recent studies suggested that SCIN participates in the development and progression of several human cancers. SCIN is highly expressed in both human prostate cancer specimens and in the PC3 prostate cancer cell line, and suppression of SCIN results in decreased cell proliferation and G0/G1 phase arrest [16]. High SCIN expression has also been observed in human lung cancer specimens, and the level of SCIN is associated with cell proliferation in human lung carcinoma cell lines [17]. A quantitative proteomic analysis showed that SCIN was a cisplatin-resistant marker in the HT1376 human bladder cancer cell line [18], and SCIN knockdown effectively reduces cell proliferation and migration in the SGC7901 human GC cell line [19]. However, the clinical significance and the precise mechanisms that control SCIN expression in cancer have not been established.

In the present study, we report that SCIN is overexpressed in human GC tissues and the expression level correlates with the tumor invasion depth, lymph node metastasis and poor overall survival of patients. SCIN knockdown impaired the invasion and 
metastasis of GC cells, accompanied by decreased filopodium formation and Cdc42 expression. Our results suggest that SCIN may be considered as a novel prognostic indicator and a therapeutic target for human GC.

\section{Material and Methods}

\subsection{Patients and tissue samples}

A total of 188 formalin-fixed and paraffin-embedded surgical specimens of GC tumors and paired adjacent normal tissues were collected. All patients were enrolled in the Southwest Hospital, Chongqing, China, from 2006 to 2007 and had not received radiotherapy or chemotherapy before radical gastrectomy. Follow-up information was available for all patients. Each GC specimen was histologically graded by two pathologists according to the World Health Organization (WHO) standard and TNM staging (Union for International Cancer Control [20]) in a double-blinded manner. Written informed consent was obtained from all patients. The study was approved by the Ethical Committee of Southwest Hospital. The detailed information of patients and IHC scores was summarized in Table S1 and listed in the file named "clinical information" in the supplementary file.

\subsection{Cell culture}

Human GC cell line MGC803 (Cell Bank of Shanghai Institute of Cell Biology, Chinese Academy of Sciences) and primary GC cells XN0422 established by our 
laboratory were cultured in RPMI 1640 medium (Sigma, USA) supplemented with $10 \%$ fetal bovine serum (FBS) (Gibco, USA) at $37^{\circ} \mathrm{C}$ in $5 \% \mathrm{CO}_{2}$ and $100 \%$ humidity.

\subsection{Immunohistochemistry (IHC)}

Immunohistochemical staining was performed on $4 \mu \mathrm{m}$ tissue sections using a Dako REAL Envision Detection System (Dako, Glostrup, Denmark) according to the manufacturer's instructions. After antigen retrieval and blocking, the tissue slides were incubated with a mouse anti-SCIN monoclonal antibody (1:50, Santa Cruz, USA) at $4^{\circ} \mathrm{C}$ overnight. After removing the primary antibody with PBS, a horseradish peroxidase (HRP)-conjugated secondary antibody (Dako) was added, and the slides were incubated at $37^{\circ} \mathrm{C}$ for $30 \mathrm{~min}$. All slides were evaluated independently by two pathologists in a blinded manner. An IHC score was obtained by multiplying the staining intensity from the average percentage of positive cells, following an accepted semi-quantitative evaluation method [21]. The staining intensity was scored as follows: 0 , no staining; 1 , weak staining; 2 , moderate staining; and 3, strong staining (Fig. S1). The percentage of positive cancer cells was scored as: $1=1-25 \% ; 2=$ $26-50 \% ; 3=51-75 \%$; and $4>75 \%$. Scores were analyzed using the statistical software X-tile with a score of 2 as the cutoff value [22]. Total scores $\geq 2$ were defined as $\mathrm{SCIN}^{\text {high }}$ and scores $<2$ were defined as $\mathrm{SCIN}^{\text {low }}$. 
The construction of SCIN shRNA lentiviral particles was performed by Life Technologies Co. Ltd (Shanghai, China). Three shRNA sequences targeting SCIN and a non-targeting scrambled sequence were listed in Table S2. MGC803 and XN0422 cells were cultured in 24-well plates and infected with lentivirus containing SCIN shRNA (sh-SCIN) or scrambled shRNA (mock) at $3 \times 10^{6} /$ well for $24 \mathrm{~h}$ without FBS. Fresh culture medium containing $3 \mu \mathrm{g} / \mathrm{mL}$ puromycin was added to select stable puromycin-resistant cells. The knockdown efficiency of SCIN was confirmed by RT-PCR and western blotting (Fig. S2).

\subsection{RNA extraction and quantitative real-time PCR ( $q R T-P C R)$}

Total RNA was extracted from GC cells using Trizol reagent (Takara, Japan), and reverse-transcription was performed using reverse transcription reagents (Takara, Japan). qRT-PCR reactions were performed using a CFX96 Real-Time PCR Detection System (Bio-Rad) with SYBR Premix Ex Taq II (Takara) following the manufacturer's instructions. The primers used in this study were listed in Table S3. The relative mRNA expression levels were determined by the cycle threshold $(\mathrm{Ct})$ normalized against $\beta$-actin using the $2^{-\Delta \Delta \mathrm{Ct}}$ formula.

\subsection{Western blotting}

GC cells were lysed with radioimmunoprecipitation assay (RIPA) Lysis Buffer 
(Beyotime Institute of Biotechnology, China) supplemented with protease inhibitors (PMSF, Thermo, USA). The lysate was centrifuged for $15 \mathrm{~min}$ at $15,000 \mathrm{~g}$ and $4{ }^{\circ} \mathrm{C}$. The proteins in the supernatant were separated by $10 \%$ SDS-PAGE and transferred onto polyvinylidene fluoride (PVDF) membranes (Millipore, USA). The membranes were blocked with $5 \%$ evaporated milk for $2 \mathrm{~h}$ and subsequently incubated with a mouse anti-human SCIN monoclonal antibody (1:1000, Santa Cruz, USA), a mouse anti-human Cdc42 monoclonal antibody (1:1000, Cytoskeleton, Denver, CO, USA), or a mouse anti-human $\beta$-actin monoclonal antibody (1:1000, Beyotime, China) overnight at $4^{\circ} \mathrm{C}$. The membranes were then incubated with the appropriate HRP-conjugated secondary antibody for $2 \mathrm{~h}$ at room temperature. Chemiluminescence was detected using SuperSignal West Femto Maximum Sensitivity Substrate (ECL, Pierce-Thermo Scientific, USA). $\beta$-actin was used as a loading control.

\subsection{In vitro invasion assay}

Transwells ( $8 \mu \mathrm{m}, 24$-well format) (BD Falcon, USA) were coated with $10 \mu \mathrm{L}$ of Matrigel (BD, USA) / RPMI-1640 (1:1, v/v) for $30 \mathrm{~min}$ at $37^{\circ} \mathrm{C}$. A total of $2 \times 10^{4}$ cells were seeded into the upper chambers in $200 \mu \mathrm{L}$ serum-free RPMI-1640 medium. The lower chambers were filled with $500 \mu \mathrm{L}$ RPMI-1640 medium containing $10 \%$ FBS. Following $24 \mathrm{~h}$ of incubation, the membranes were fixed with $4 \%$ paraformaldehyde for $20 \mathrm{~min}$. The cells on the upper surface of the membrane were removed with a cotton swab, and the cells on the lower surface of the membrane were stained with 
crystal violet solution (Beyotime, China). The number of invading cells was counted in five randomly selected high powered fields and photographed under a microscope.

\subsection{Cell monolayer scratching assay}

GC cells were grown in 24-well plates with RPMI-1640 medium supplemented with $10 \%$ FBS until $90 \%$ confluence. Wounds were created by scratching the cell surface with a $10 \mu \mathrm{L}$ pipette tip. After washing with PBS, fresh RPMI-1640 medium without FBS was added. Cells moving and growing across the scratched lines were monitored every hour for $24 \mathrm{~h}$ using a Live Cell Imaging System (ZEISS, Germany). The migratory ability of the cells was quantified as the gap distance recovered compared with the original gap.

\subsection{Immunofluorescence and confocal laser scanning microscopy}

For the detection of filopodia, cells were seeded on cover slides in 24-well plates in RPMI-1640 medium supplemented with $10 \%$ FBS and grown to $70 \%$ confluence. The cells were fixed in $4 \%$ paraformaldehyde for $20 \mathrm{~min}$ at room temperature and then permeabilized with $0.5 \%$ Triton X-100 for 5 min. After washing with PBS, the cells were stained with rhodamine phalloidin (Cytoskeleton, Denver, CO, USA), and nuclei were stained with Hoechst. The fluorescence of the labeled cells was visualized by inverted confocal laser microscopy (Leica, Germany). 


\subsection{Subcutaneous xenograft tumorigenicity and peritoneal dissemination in mice}

Female BALB/c nude mice (5 to 6 weeks old) were purchased from the Laboratory Animal Center of the Third Military Medical University (Chongqing, China) and housed in a pathogen-free environment. GC cells with different treatments were injected subcutaneously into the axilla of the mice $\left(2 \times 10^{4}\right.$ cells in $0.2 \mathrm{~mL}$ Matrigel $(1: 1, v / v))$. Subcutaneous tumor growth was inspected every day. The mice were sacrificed at the end of 4 weeks post-implantation, and the tumor mass was weighed. To examine the effect of SCIN on in vivo metastasis, GC cells with different treatments were injected intraperitoneally $\left(1 \times 10^{5}\right.$ cells per mouse). After 6 weeks, the mice were sacrificed and the number of intraperitoneal tumor foci was counted. All animal procedures were approved by the Third Military Medical University Animal Committee.

\subsection{Statistical analyses}

All experiments were conducted at least three times, and the results shown are from representative experiments. Data are presented as the mean \pm SD. Statistical analysis was performed using the SPSS 17.0 software (SPSS, Inc., Chicago, IL, USA). Student's $t$ test was used for comparing two groups. The Pearson $\chi^{2}$ test was used to determine the correlation between SCIN expression and the clinicopathological features of GC patients. Kaplan-Meier survival plots and log-rank statistics were used 
to compare the survival rates of patients. Univariate and multivariate survival analyses were performed using a Cox proportional-hazards model with stepwise selection. The cutoff value of the IHC score was determined by relative risk analysis with the statistical software X-tile [22] and Youden's index calculated by SPSS software (Fig. S3). $\mathrm{P}<0.05$ was considered significant.

\section{Results}

3.1 High SCIN expressed in GC specimens is associated with poor outcomes of patients

The expression levels of SCIN in tumor tissues, paired adjacent normal tissues, and metastatic lymph nodes of GC patients were examined using IHC. SCIN staining was observed in the cytoplasm and membranes of GC cells (Fig. 1A). The expression of SCIN was very low or absent in normal gastric mucosa (Fig. 1Aa), and the proportion of SCIN ${ }^{\text {high }}$ cells in GC tissues $(56 \%, 105 / 188)$ was markedly higher than that in the paired adjacent normal tissues $(5.9 \%, 11 / 188)(p=0.000)$ (Fig. 1B, Table 1). As shown in Fig. 1Ab-f, the levels of SCIN expression in GC tumors were positively correlated with the invasive depth $(p=0.011)$ and lymph node metastasis $(p=0.024)$, but not with the histological grade $(p=0.483)$ (Table 2).

We next analyzed the association of SCIN expression with the lifespan of GC patients after surgery. Kaplan-Meier survival curves showed that the overall survival rates for 
patients with $\mathrm{SCIN}^{\text {low }}$ GC were significantly higher than those with SCIN $^{\text {high }}$ tumors (Fig. 1C, $p<0.001$ ). Univariate and multivariate analyses showed that the expression of SCIN was an independent prognostic indicator for the overall survival of GC patients (Table 3).

3.2 SCIN depletion reduces the migration and invasion of GC cells in vitro

To examine the effect of SCIN on the migration and invasion of GC cells, in vitro cell invasion and scratch wound assays were performed after SCIN-knockdown in MGC803 and XN0422 cells. As shown in Fig. 2A and B, GC cells with SCIN-knockdown migrated into the scratching area more slowly than control cells ( $p$ $<0.05)$. GC cells with SCIN-knockdown also exhibited decreased invasive capability ( $p<0.05$, Fig. $2 \mathrm{C}$ and D). These results suggest that SCIN contributes to the migratory and invasive abilities of GC cells in vitro.

3.3 Suppression of SCIN attenuates the tumorigenic capability and metastasis of GC cells in vivo

We then assessed the effect of SCIN knockdown on the tumorigenic potential of MGC803 and XN0422 GC cells using a subcutaneous xenograft model in nude mice. Although all cells, including SCIN knockdown and mock cells, possessed the capability to form tumors in nude mice when injected at $2 \times 10^{4}$ cells/mouse, the size of the xenograft tumors derived from the SCIN knockdown MGC803 and XN0422 
cells were significantly smaller than those formed by mock-transfected cells (Fig. 3A). The weights of the xenograft tumors corresponded to their sizes (Fig. 3A and B) $(p<$ 0.001). The GC nature of xenograft tumors was verified by hematoxylin \& eosin (H\&E) staining of tumor sections (Fig. 3C). The xenograft tumors derived from the mock MGC803 and XN0422 cells appeared as early as two weeks post-implantation, whereas no subcutaneous tumors were observed in mice injected with the SCIN knockdown GC cells at the same time point. To examine the role of SCIN in the metastasis of GC cells, SCIN knockdown (sh-SCIN3) MGC803 or XN0422 cells or mock-transfected cells were injected into the peritoneal cavity of nude mice $(n=6)$. Although disseminated tumors were found in the four mouse groups at 6 weeks post-implantation, fewer metastatic tumors were observed in mice implanted with SCIN-knockdown GC cells compared to mice implanted with mock-transfected GC cells (Fig. 3D and E). Thus, SCIN plays an important role in GC tumorigenesis, invasion and dissemination.

\subsection{Knockdown of SCIN inhibits Cdc42-mediated filopodium formation by GC cells}

The migration of tumor cells, which involves remodeling of the actin cytoskeleton during the epithelial to mesenchymal transition, is an essential factor in metastasis [23]. To successfully metastasize, cancer cells must alter their cytoskeleton to adapt to and survive in the surrounding microenvironments [24]. Filopodia are actin-based protrusions that promote cell migration and invasion [25-26]. Because SCIN is an 
actin severing and capping protein that regulates the actin cytoskeleton, we postulated that SCIN might affect the formation of filopodia. We therefore examined morphological changes in the filopodia of wild-type, mock and SCIN knockdown MGC803 and XN0422 cells. Phalloidine staining examined under confocal microscopy showed that the filopodia of both SCIN-knockdown GC cell groups were markedly impaired compared with the wild type and mock cells (Fig. 4A). We further addressed whether Cdc42, a regulator of filopodia [27], is involved in the SCIN-mediated regulatory of filopodium formation in GC cells. The mRNA expression of Cdc42 in both SCIN knockdown GC cell groups was down-regulated by 50 percent compared with the wild-type and mock MGC803 and XN0422 cells (Fig. 4B). Knockdown of SCIN resulted in significant decrease in the expression of Cdc42 protein in both groups of GC cells (Fig. 4C). Interestingly, compared to the mRNA, Cdc42 protein was more significantly down-regulated in both types of GC cells with GC knockdown. Thus, SCIN appears to regulate the expression of Cdc42 in GC cells at both mRNA and protein levels.

\section{Discussion}

Cancer cell metastasis is a multi-stage process involving invasion into surrounding tissues, intravasation, transit in the blood or lymph, extravasation, and growth at distant sites. These processes require increased cell motility driven by remodeling of the actin cytoskeleton [28-29]. Increasing evidence suggests that aberrant actin 
cytoskeleton remodeling is associated with abnormalities of cytoskeleton-associated proteins, which regulate actin polymerization and depolymerization in various ways, such as increasing the rate of monomer addition to barbed ends, nucleating new filaments, increasing the number of barbed ends, and reducing depolymerization [30-31]. As an important actin-binding protein, SCIN participates in actin-capping, -severing and -nucleating. It has been reported that knockdown of SCIN decreases the migratory capability of the highly metastatic GC cell line SGC7901 by promoting the epithelial-mesenchymal transition (EMT) [19]. In the present study, we found that SCIN expression was correlated with the invasion depth and lymph node metastasis of human GC, and associated with decreased survival of patients. To our knowledge, this is the first clinicopathological study to link SCIN to the outcome of the surgical treatment of GC.

To establish a motile and invasive phenotype, cancer cells develop unique morphogenic transition programs. Following actin cytoskeleton reorganization, membrane protrusions are formed, including lamellipodia, filopodia, podosomes, and invadopodia [25, 32-34]. Filopodia are slender cytoplasmic projections consisting of 10 or more tightly bundled parallel actin filaments [35] that extend beyond the leading edge of the membrane in migratory cells, functioning as antennae for probing the surrounding environment [25] to facilitate cell migration. Moreover, filopodia function in a number of other cellular processes including wound healing, adhesion to the extracellular matrix, guidance toward chemoattractants, neuronal growth-cone pathfinding, and embryonic development [36-37]. The actin filaments of filopodia are 
regulated by actin-binding proteins with their barbed ends facing toward the plasma membrane [38], and an increase in the density of filopodia has been viewed as a characteristic of invasive cancer cells [39]. In osteosarcoma, in vivo metastasis and in vitro cell migration were suppressed when filopodium formation was inhibited [40], and increased formation of filopodia was found to enhance the migration of breast cancer cells [41]. We observed that filopodium formation by GC cells was inhibited by knockdown of SCIN, resulting in attenuated metastatic and migratory capabilities.

As a key regulator of filopodia, the aberrant expression of Cdc42 may lead to disorders in filopodia. Indeed, a previous study indicated that expression of a dominant-negative form of $\mathrm{Cdc} 42$ in NIH $3 \mathrm{~T} 3$ cells impaired cell spreading by inhibiting filopodium formation [42]. Due to the unique role of Cdc42 in filopodium formation, we assessed the effect of SCIN on Cdc42 expression in GC cells. Interestingly, the expression of Cdc42 was impaired following SCIN knockdown in GC cells, resulting in deficient filopodium formation. This finding suggests that SCIN enhances the invasive and metastatic potential of GC cells by stimulating the formation of filopodia via a Cdc42 pathway.

In conclusion, our study illustrates an important function of SCIN in the malignant behavior of GC. We demonstrate that high levels of SCIN expression in GC tissue correlate with poor prognosis of patients. Furthermore, SCIN enhances the invasion and metastasis of GC cells through activating the Cdc42 pathway to increase the formation of filopodia. Thus, SCIN is a potential clinical biomarker to predict the outcome and a therapeutic target for human GC. 


\section{Acknowledgments}

This project was supported by grants from the National Science Foundation of China (Nos. 81272365 and 81372555). We thank Prof. Pei-wu Yu (Institute of Pathology and

Southwest Cancer Center, Southwest Hospital, Third Military Medical University, Chongqing, China) for supporting this work. We thank Mrs. Ze-xuan Yan (Institute of Pathology and Southwest Cancer Center, Southwest Hospital, Third Military Medical University, Chongqing, China) for technical assistance in cell culture.

\section{Conflicts of interest:}

The authors declare no conflicts of interest.

\section{References}

[1] A. Jemal, F. Bray, M.M. Center, J. Ferlay, E. Ward, D. Forman, Global cancer statistics, CA Cancer J. Clin. 61 (2011) 69-90.

[2] B. Vogelstein, N. Papadopoulos, V.E. Velculescu, S. Zhou, L.A. Diaz, K.W. Kinzler, Cancer genome landscapes, Science 339 (2013) 1546-1558.

[3] R. Wadhwa, S. Song, J. Lee, Y. Yao, Q. Wei, J.A. Ajani, Gastric cancer - molecular and clinical dimensions, Nat. Rev. Clin. Oncol. 10 (2013) 643-655.

[4] G.H. Li, P.D. Arora, Y. Chen, C.A. McCulloch, P. Liu, Multifunctional roles of gelsolin in health and diseases, Med. Res. Rev. 32 (2012) 999-1025. 
[5] M. Tanaka, L. Mullauer, Y. Ogiso, Fujita H, Moriya S, Furuuchi K, et al, Gelsolin: a candidate for suppressor of human bladder cancer, Cancer Res. 55 (1995) 32283232.

[6] M. Yilmaz, and G. Christofori, Mechanisms of motility in metastasizing cells. Mol Cancer Res 8 (2010) 629-42.

[7] D.J. Kwiatkowski, Functions of gelsolin: motility, signaling, apoptosis, cancer, Curr. Opin. Cell Biol. 11 (1999) 103-108.

[8] D.C.A. Rodriguez, M.L. Vitale, L. Tchakarov, J.M. Trifaro, Human platelets contain scinderin, a $\mathrm{Ca}(2+)$-dependent actin filament-severing protein, Thromb. Haemost. 67 (1992) 248-251.

[9] P.T. Dumitrescu, S.D. Rose, T. Lejen, M.G. Marcu, J. Trifaro, Expression of various scinderin domains in chromaffin cells indicates that this protein acts as a molecular switch in the control of actin filament dynamics and exocytosis, $\mathrm{J}$. Neurochem. 92 (2005) 780-789.

[10] J.- Trifaró, S. Gasman, L.M. Gutiérrez, Cytoskeletal control of vesicle transport and exocytosis in chromaffin cells, Acta Physiol. 192 (2008) 165-172.

[11] H. Jiang, Y. Wang, A. Viniegra, C. Sima, C.A. McCulloch, M. Glogauer, Adseverin plays a role in osteoclast differentiation and periodontal disease-mediated bone loss, FASEB J. 29 (2015) 2281-2291.

[12] S. Hassanpour, H. Jiang, Y. Wang, J.W.P. Kuiper, M. Glogauer, The actin binding protein adseverin regulates osteoclastogenesis, PLOS ONE 9 (2014) e109078.

[13] D. Nurminsky, C. Magee, L. Faverman, M. Nurminskaya, Regulation of 
chondrocyte differentiation by actin-severing protein adseverin, Dev. Biol. 302 (2007) 427-437.

[14] R. Zunino, Q. Li, S.D. Rose, Expression of scinderin in megakaryoblastic leukemia cells induces differentiation, maturation, and apoptosis with release of plateletlike particles and inhibits proliferation and tumorigenesis, Blood 98 (2001) 2210-2219.

[15] X. Li, H. Jiang, Y. Huang, Q. Gong, J. Wang, J. Ling, Expression and function of the actin-severing protein adseverin in the proliferation, migration, and differentiation of dental pulp cells, J. Endod. 41 (2015) 493-500.

[16] D. Wang, S.Q. Sun, Y.H. Yu, W.Z. Wu, S.L. Yang, J.M. Tan, Suppression of SCIN inhibits human prostate cancer cell proliferation and induces G0/G1 phase arrest, Int. J. Oncol. 44 (2014) 161-166.

[17] H. Liu, D. Shi, T. Liu, Z. Yu, C. Zhou, Lentivirus-mediated silencing of SCIN inhibits proliferation of human lung carcinoma cells, Gene 554 (2015) 32-39.

[18] N. Miura, N. Takemori, T. Kikugawa, N. Tanji, S. Higashiyama, M. Yokoyama, Adseverin: a novel cisplatin-resistant marker in the human bladder cancer cell line HT1376 identified by quantitative proteomic analysis, Mol. Oncol. 6 (2012) 311-322. [19] X.M. Chen, J.M. Guo, P. Chen, Mao LG, Feng WY, Le DH, et al, Suppression of scinderin modulates epithelial-mesenchymal transition markers in highly metastatic gastric cancer cell line SGC-7901, Mol. Med. Rep. 10 (2014) 2327-2333.

[20] K. Washington, K. Washington, 7th edition of the AJCC cancer staging manual: stomach, Ann. Surg. Oncol. 17 (2010) 3077-3079. 
[21] H. Shi, S. Chen, H. Jin, C. Xu, G. Dong, Q. Zhao, et al., Downregulation of MSP58 inhibits growth of human colorectal cancer cells via regulation of the cyclin D1-cyclin-dependent kinase 4-p21 pathway, Cancer Sci. 100 (2009) 1585-1590.

[22] R.L. Camp, M. Dolled-Filhart, D.L. Rimm, X-tile: a new bio-informatics tool for biomarker assessment and outcome-based cut-point optimization, Clin. Cancer Res. 10 (2004) 7252-7259.

[23] V. Vasioukhin, C. Bauer, M. Yin, E. Fuchs, Directed actin polymerization is the driving force for epithelial cell-cell adhesion, Cell 100 (2000) 209-219.

[24] M. Raftopoulou, A. Hall, Cell migration: Rho GTPases lead the way, Dev. Biol. 265 (2004) 23-32.

[25] P.K. Mattila, P. Lappalainen, Filopodia: molecular architecture and cellular functions, Nat. Rev. Mol. Cell Biol. 9 (2008) 446-454.

[26] A. Nürnberg, T. Kitzing, R. Grosse, Nucleating actin for invasion, Nat. Rev. Cancer 11 (2011) 177-187.

[27] P. Collart-Dutilleul, I. Panayotov, E. Secret, F. Cunin, C. Gergely, F. Cuisinier, et al., Initial stem cell adhesion on porous silicon surface: molecular architecture of actin cytoskeleton and filopodial growth, Nanoscale Res. Lett. 9 (2014) 564.

[28] T. D. Pollard, and G. G. Borisy, Cellular motility driven by assembly and disassembly of actin filaments. Cell 112 (2003) 453-65.

[29] S.M. Rafelski, and J.A. Theriot, Crawling toward a unified model of cell mobility: spatial and temporal regulation of actin dynamics. Annu Rev Biochem 73 (2004) 209-39. 
[30] S.D. Bernal, and R.A. Stahel, Cytoskeleton-associated proteins: their role as cellular integrators in the neoplastic process. Crit Rev Oncol Hematol 3 (1985) 191-204.

[31] M.F. Olson, and E. Sahai, The actin cytoskeleton in cancer cell motility. Clin Exp Metastasis 26 (2009) 273-87.

[32] A. Wicki, F. Lehembre, N. Wick, B. Hantusch, D. Kerjaschki, and G. Christofori, Tumor invasion in the absence of epithelial-mesenchymal transition: podoplanin-mediated remodeling of the actin cytoskeleton. Cancer Cell 9 (2006) 261-72.

[33] B. Blouw, D.F. Seals, I. Pass, B. Diaz, and S.A. Courtneidge, A role for the podosome/invadopodia scaffold protein Tks5 in tumor growth in vivo. Eur J Cell Biol 87 (2008) 555-67.

[34] A. Bergman, J.S. Condeelis, and B. Gligorijevic, Invadopodia in context. Cell Adh Migr 8 (2014) 273-9.

[35] D. Vignjevic, S. Kojima, Y. Aratyn, O. Danciu, T. Svitkina, G.G. Borisy, Role of fascin in filopodial protrusion, J. Cell Biol. 174 (2006) 863-875.

[36] S.L. Gupton, F.B. Gertler, Filopodia: the fingers that do the walking, Sci. STKE 2007 (2007) e5.

[37] J. Faix, K. Rottner, The making of filopodia, Curr. Opin. Cell Biol. 18 (2006) $18-25$.

[38] C. Wu, S. Asokan, M. Berginski, E. Haynes, N. Sharpless, J. Griffith, et al., Arp2/3 is critical for lamellipodia and response to extracellular matrix cues but is dispensable for chemotaxis, Cell 148 (2012) 973-987. 
[39] D. Vignjevic, M. Schoumacher, N. Gavert, K.- Janssen, G. Jih, M. Lae, et al., Fascin, a novel target of beta-catenin-TCF signaling, is expressed at the invasive front of human colon cancer, Cancer Res. 67 (2007) 6844-6853.

[40] N. Agarwal, A.S. Adhikari, S.V. Iyer, K. Hekmatdoost, D.R. Welch, T. Iwakuma, MTBP suppresses cell migration and filopodia formation by inhibiting ACTN4, Oncogene 32 (2013) 462-470.

[41] Y. Li, Z. Zhang, X. Zhou, L. Li, Q. Liu, Z. Wang, et al., The oncoprotein HBXIP enhances migration of breast cancer cells through increasing filopodia formation involving MEKK2/ERK1/2/Capn4 signaling, Cancer Lett. 355 (2014) 288-296. [42] H. Guillou, A. Depraz-Depland, E. Planus, B. Vianay, J. Chaussy, A. Grichine, et al., Lamellipodia nucleation by filopodia depends on integrin occupancy and downstream Rac1 signaling, Exp. Cell Res. 314 (2008) 478-488. 
Table 1. SCIN expression in GC and adjacent normal mucosa

\begin{tabular}{lccc}
\hline & SCIN $^{\text {low }}$ & SCIN $^{\text {high }}$ & P-value \\
\hline Carcinoma & 83 & 105 & $<0.0001$ \\
Adjacent normal mucosa & 177 & 11 & \\
\hline
\end{tabular}


Table 2. The relationship between SCIN expression and clinicopathological features of GC patients

\begin{tabular}{|c|c|c|c|c|c|}
\hline \multirow[t]{2}{*}{ Prognostic variables } & \multirow[b]{2}{*}{$\mathrm{N}$} & \multicolumn{2}{|c|}{ SCIN } & \multirow[b]{2}{*}{$\chi^{2}$} & \multirow[b]{2}{*}{ P value } \\
\hline & & Low & High & & \\
\hline Age (years) & & & & 0.041 & 0.840 \\
\hline$\geq 60$ & 74 & 32 & 42 & & \\
\hline$<60$ & 114 & 51 & 63 & & \\
\hline Gender & & & & 1.502 & 0.220 \\
\hline Male & 131 & 54 & 77 & & \\
\hline Female & 57 & 29 & 28 & & \\
\hline Histological grade & & & & 0.491 & 0.483 \\
\hline $\mathrm{G} 1+\mathrm{G} 2$ & 54 & 26 & 28 & & \\
\hline G3 & 134 & 57 & 77 & & \\
\hline T stage & & & & 6.408 & 0.011 \\
\hline Tis-T2 & 61 & 35 & 26 & & \\
\hline $\mathrm{T} 3-\mathrm{T} 4$ & 127 & 48 & 79 & & \\
\hline $\mathrm{N}$ stage & & & & 5.084 & 0.024 \\
\hline No & 78 & 42 & 36 & & \\
\hline $\mathrm{N} 1-\mathrm{N} 3$ & 110 & 41 & 69 & & \\
\hline
\end{tabular}


Table 3. Univariate and multivariate analyses of overall survival in GC patients

\begin{tabular}{llllll}
\hline \multirow{2}{*}{$\begin{array}{l}\text { Prognostic } \\
\text { variables }\end{array}$} & \multicolumn{2}{c}{ Univariate analysis } & & & \multicolumn{2}{c}{ Multivariate analysis } \\
\cline { 2 - 3 } \cline { 6 - 7 } Age (years) & HR $(95 \% \mathrm{CI})$ & P value & & HR $(95 \% \mathrm{CI})$ & P value \\
Gender & $1.390(0.959-2.014)$ & 0.082 & & $1.321(0.898-1.944)$ & 0.158 \\
Histological grade & $0.742(0.501-1.097)$ & 0.135 & & $0.715(0.481-1.062)$ & 0.097 \\
T stage & $2.257(1.471-3.463)$ & 0.000 & & $1.704(1.065-2.728)$ & 0.026 \\
N stage & $2.066(1.399-3.052)$ & 0.000 & & $1.481(0.967-2.269)$ & 0.071 \\
SCIN expression & $2.182(1.477-3.223)$ & 0.000 & & $2.509(1.383-3.065)$ & 0.000 \\
\hline
\end{tabular}




\section{Figure Legends}

Fig. 1. SCIN expression in human GC tissues and its prognostic value in GC patients. (A) Representative images of SCIN staining (brown color) in normal gastric mucosa and GC samples at different infiltration depth. Normal gastric mucosa showing very low or absent SCIN expression (a); samples with carcinoma in situ (b); samples with submucosal invasion (c); samples with muscular invasion (d); samples with serosal invasion (e); and samples with lymphatic metastasis (f) SCIN was positively stained in both the membrane and cytoplasm of GC cells and the levels of SCIN increased with GC infiltration depth. (B) IHC scores of adjacent normal tissues and cancerous tissues in 188 paired GC specimens. (C) Kaplan-Meier analyses of 188 GC patients showing SCIN ${ }^{\text {high }}$ patients $(n=83)$ have shorter overall survival as compared with SCIN $^{\text {low }}$ patients $(\mathrm{n}=105)(P<0.001)$.

Fig. 2. SCIN knockdown reduces the migratory and invasive abilities of human GC cells. (A) Wound healing assays for WT, mock, and SCIN knockdown XN0422 and MGC803 cells. (B) Histograms showing the percentage of wound closure (MGC803 and XN0422 monolayer) calculated at the indicated time points. The means \pm SD from four independent experiments are shown. (C) Transwell assays performed using WT, mock, and two SCIN-knockdown MGC803 and XN0422 cells. (D) Histograms 
showing the number of cells migrating through the inserts. The means \pm SD from four independent experiments are shown. WT, wild type; mock, cells infected with mock lentivirus. ${ }^{*} P<0.05,{ }^{* \star} P<0.01,{ }^{* \star *} P<0.001$.

Fig. 3. SCIN knockdown inhibits the tumorigenicity and metastasis of GC cells. (A) Subcutaneous implantation of tumor cells in nude mice. Tumors were examined after implanting $2 \times 10^{4} \mathrm{GC}$ cells for 4 weeks. (B) Analysis of the weight of xenograft tumors. (C) H\&E staining of xenograft tumors. (D) Lower abdominal cavity tumor nodules formed by mock and sh-SCIN 3 transfected XN0422 and MGC803 cells. Arrows indicate intraperitoreal nodules. (E) Analysis of the number of intraperitoneal nodules. ${ }^{\star} P<0.05,{ }^{* *} P<0.01,{ }^{* \star} P<0.001$.

Fig. 4. Knockdown of SCIN inhibits the formation of filopodia via a Cdc42 pathway. (A) Filopodium formation observed under laser confocal scanning microscopy (600X). GC cells were stained for filopodia (red) and nuclei (blue). (B) qRT-PCR analyses of the expression of Cdc42. ${ }^{*} P<0.05$. (C) Western blotting of Cdc42 proteins. 

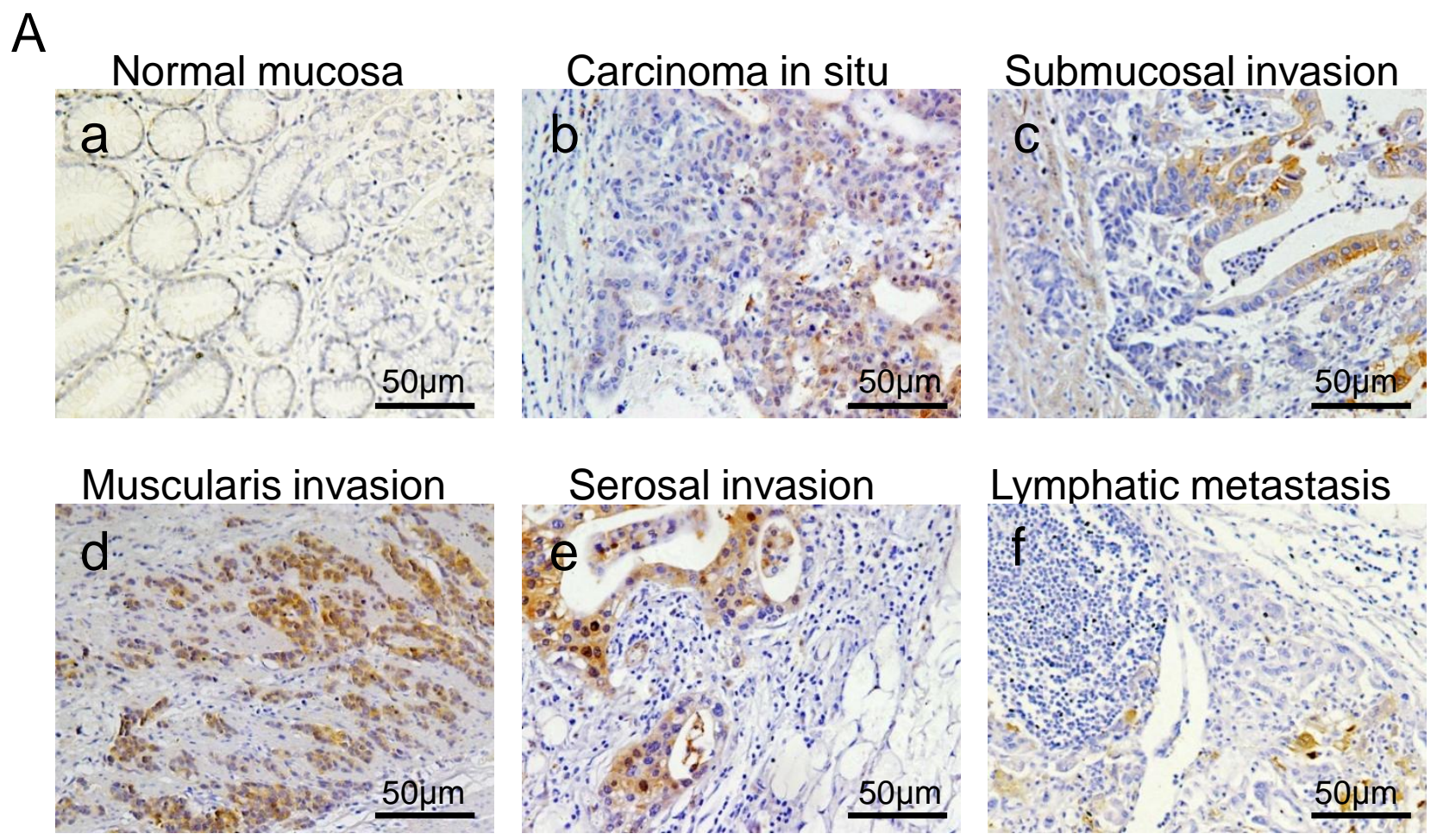

B

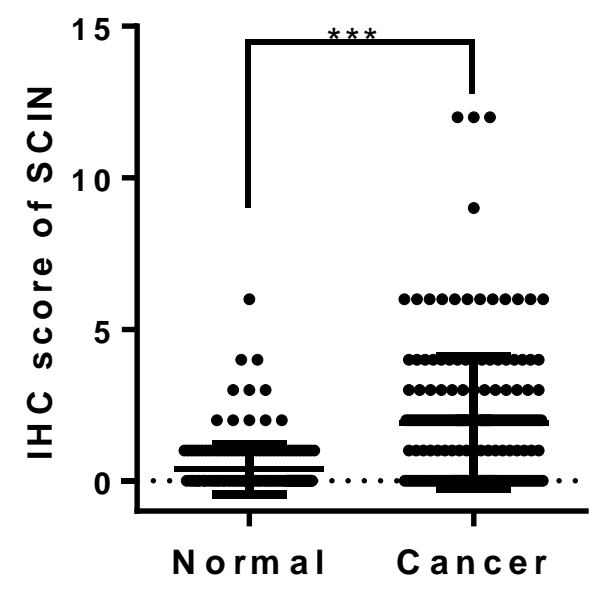

C

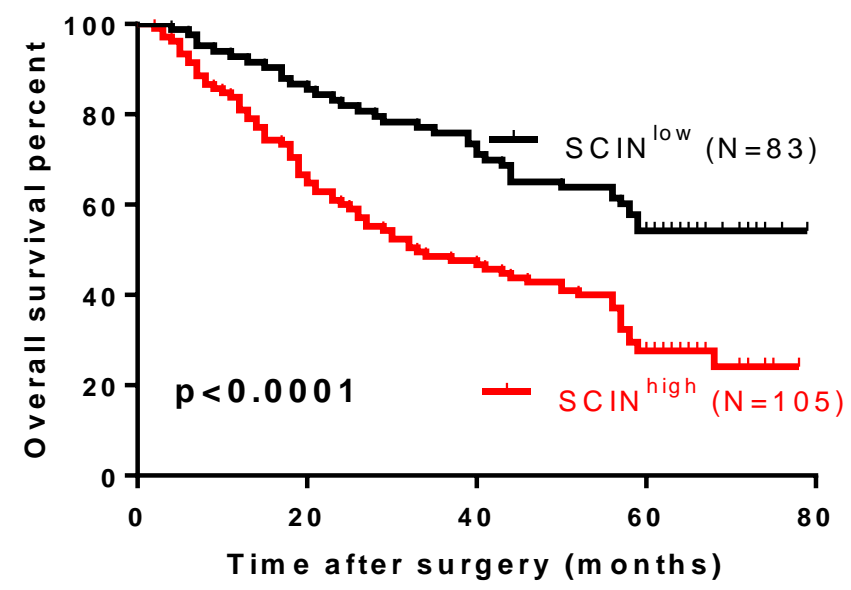

Liu J, et al. Figure 1 

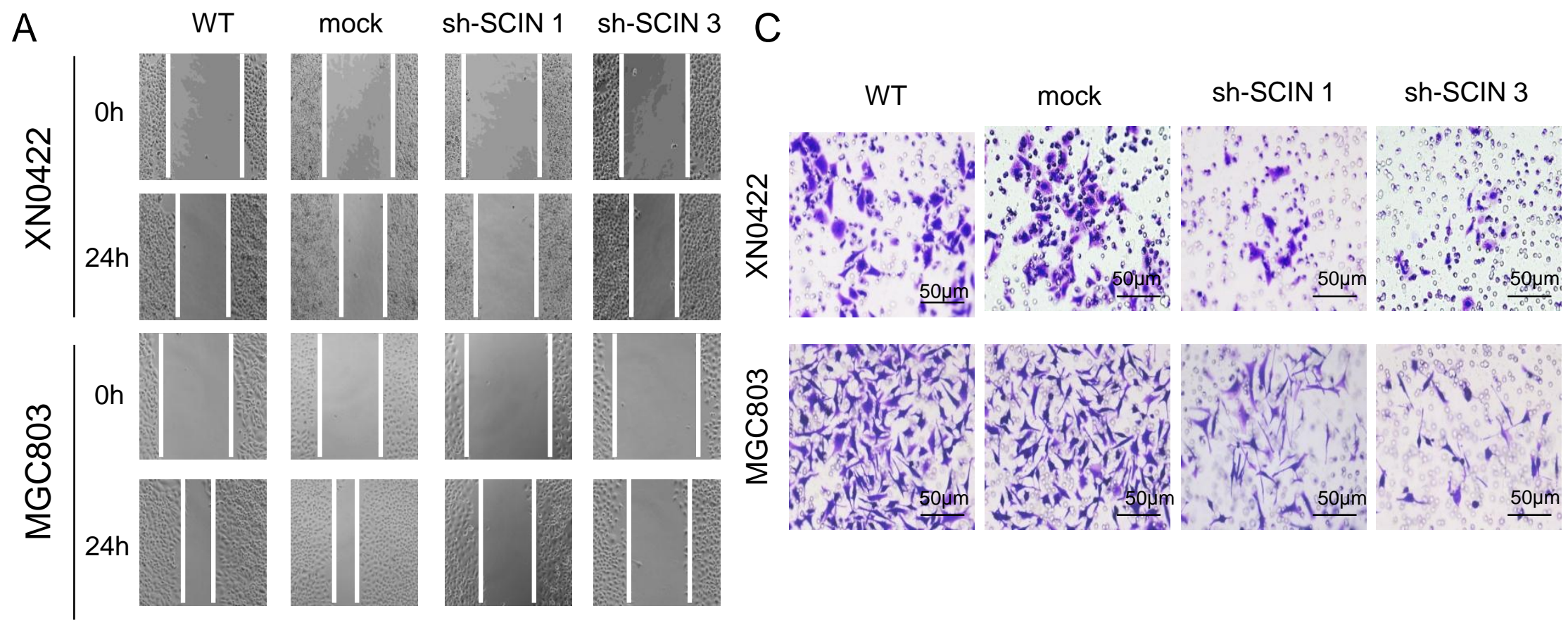

B

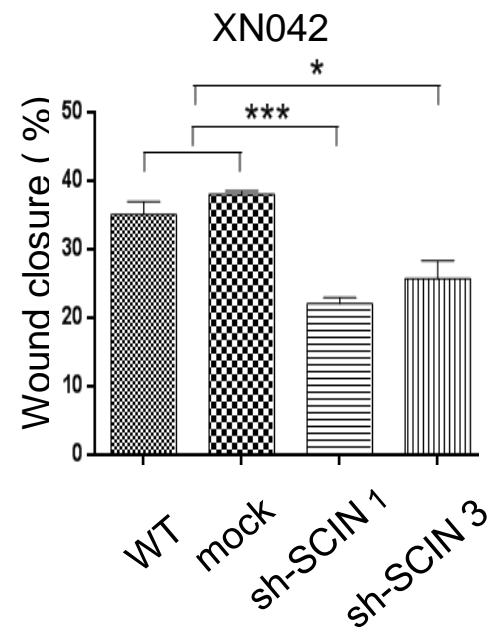

MGC803

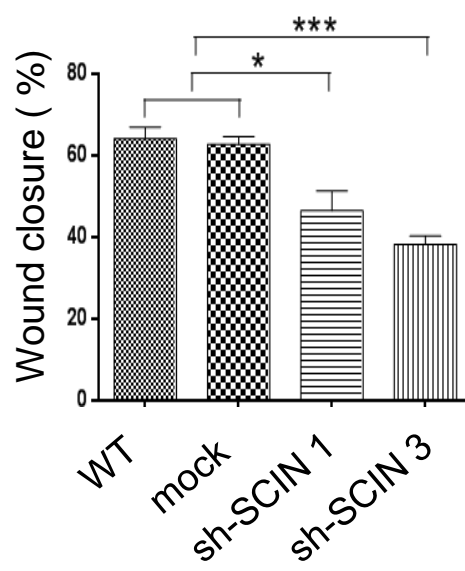

XN0422

D

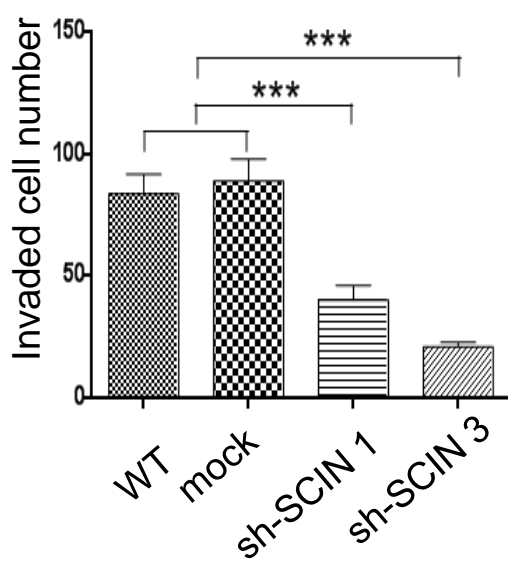

MGC803

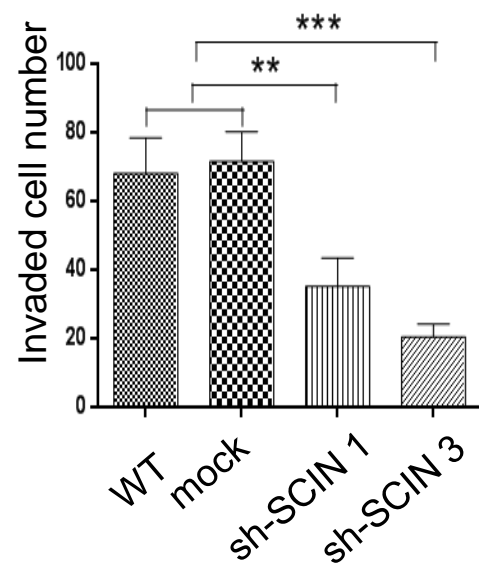

Liu J, et al. Figure 2 


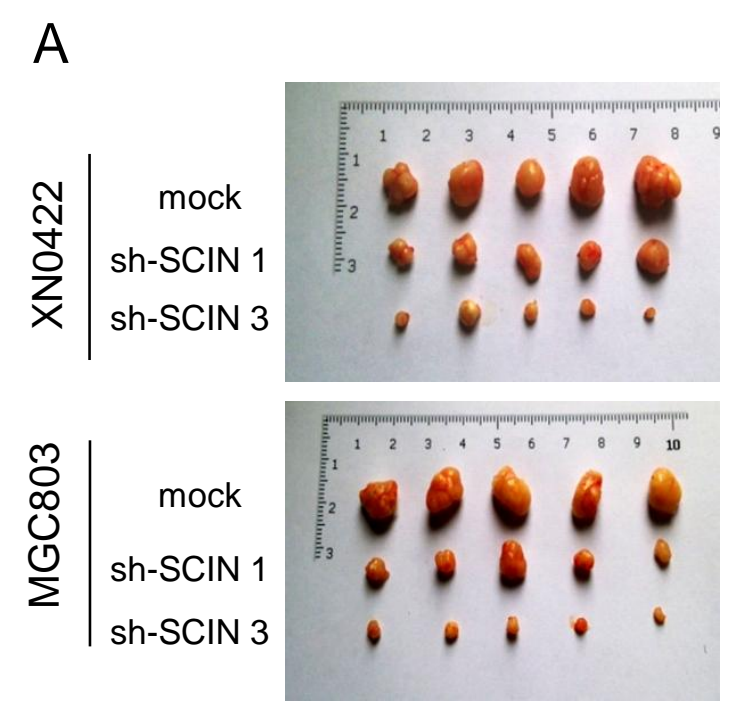

B

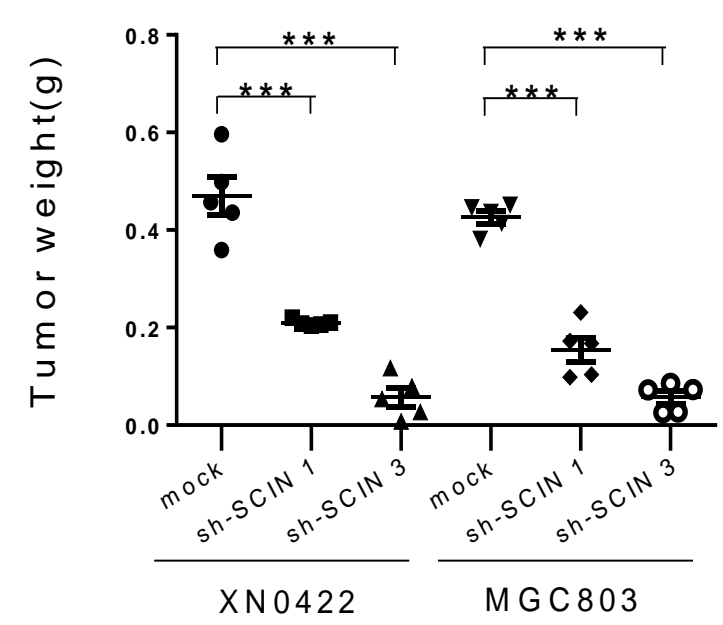

C

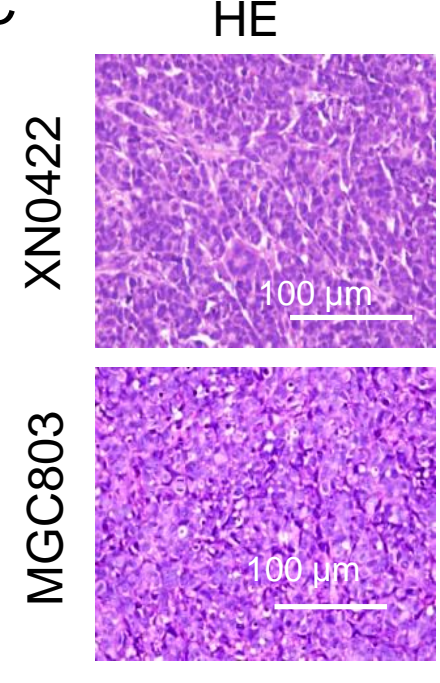

D

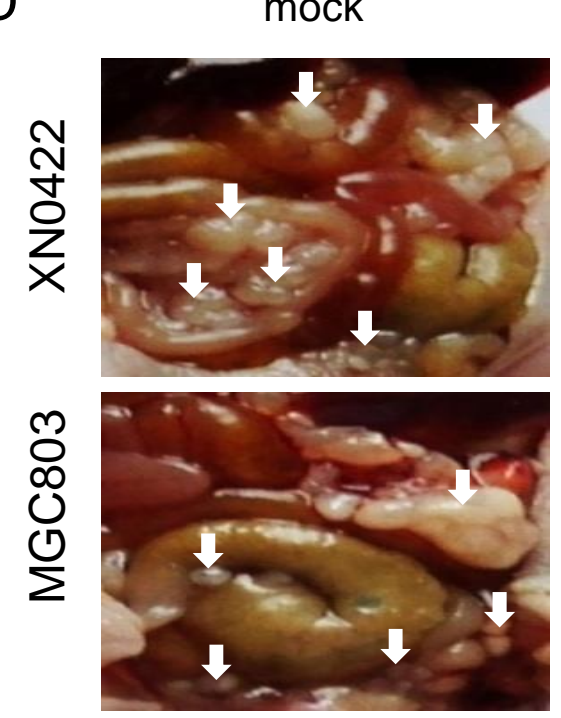

sh-SCIN 3

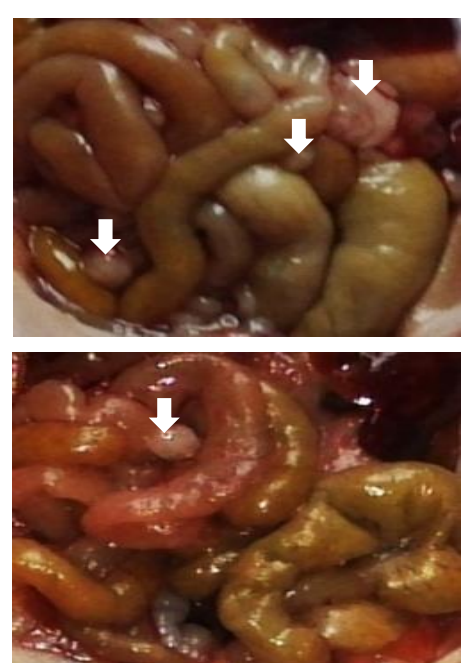

E

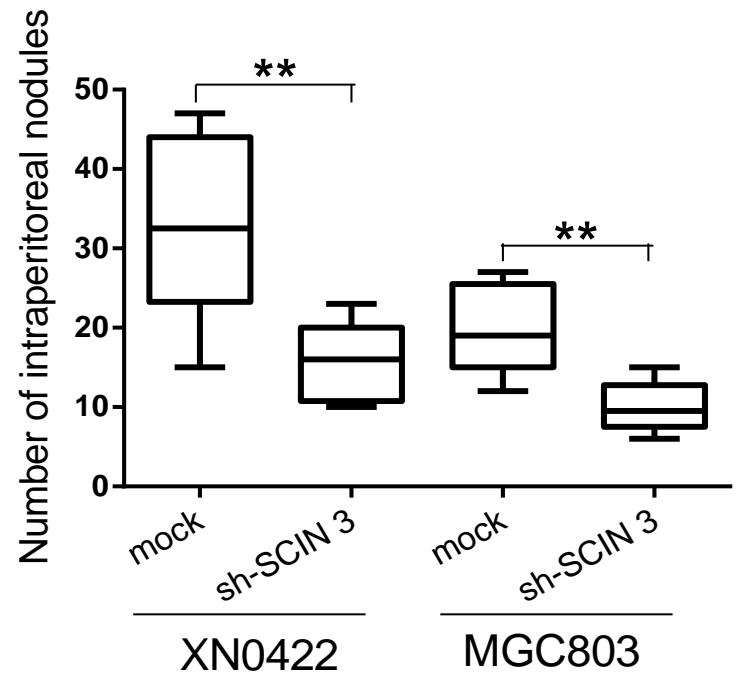

Liu J, et al. Figure 3 
A
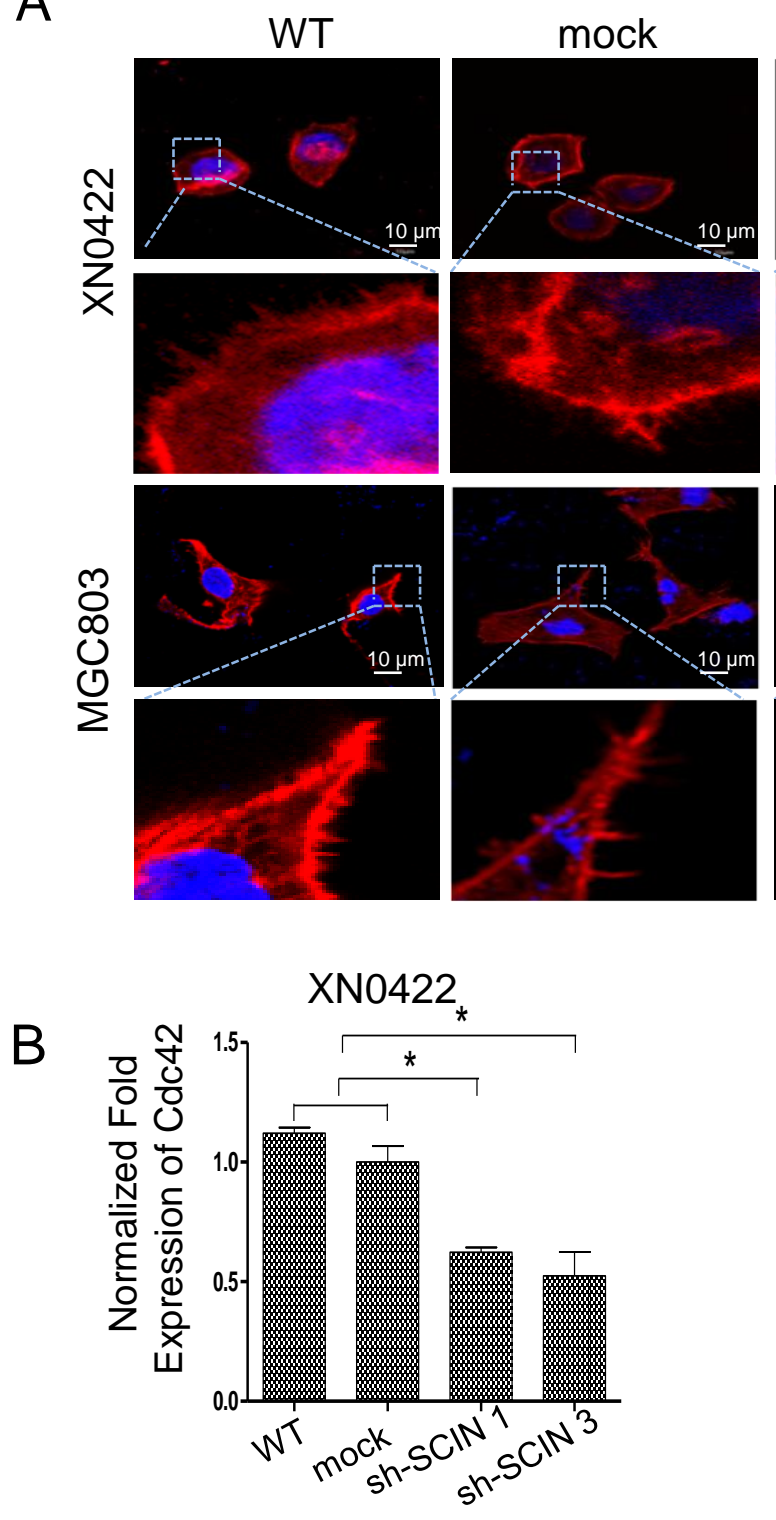

C

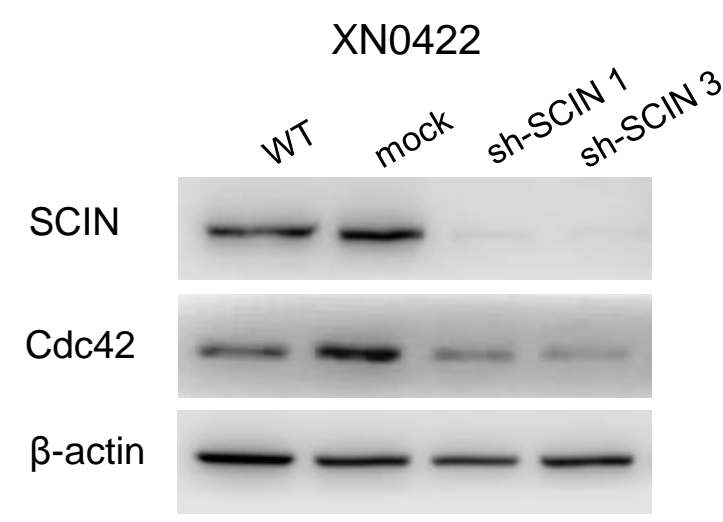

sh-SCIN 1
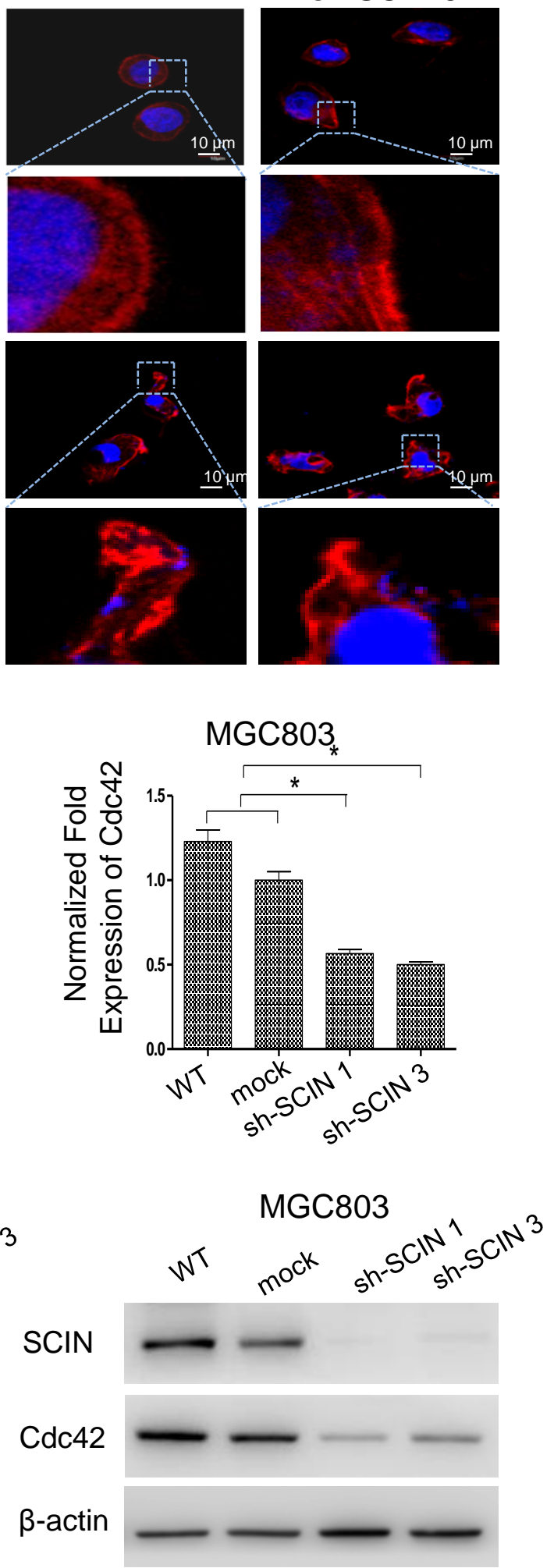